\title{
Ceftriaxone Sodium
}

National Cancer Institute

\section{Source}

National Cancer Institute. Ceftriaxone Sodium. NCI Thesaurus. Code C817.

The sodium salt form of ceftriaxone, a beta-lactam, third-generation cephalosporin antibiotic with bactericidal activity. Ceftriaxone binds to and inactivates penicillin-binding proteins (PBP) located on the inner membrane of the bacterial cell wall. PBPs participate in the terminal stages of assembling the bacterial cell wall, and in reshaping the cell wall during cell division. Inactivation of PBPs interferes with the cross-linkage of peptidoglycan chains necessary for bacterial cell wall strength and rigidity. This results in the weakening of the bacterial cell wall and causes cell lysis. Compared to the second and first generation cephalosporins, ceftriaxone is more active against gram-negative bacteria and less active against gram-positive bacteria. Ceftriaxone also crosses the blood-brain barrier and reaches therapeutic concentrations in the central nervous system (CNS). 